\title{
Explicit Solutions of the One-Dimensional Heat Equation for a Composite Wall
}

\author{
By Marcia Ascher
}

1. Introduction. Explicit numerical solutions of the equation of heat conduction in a wall of one material have been widely discussed in the literature. Consideration of the forward difference equation studied in references [2], [3], [4], and [6] suggests a variety of ways to handle the solution for a composite wall. This paper is a study of the convergence, stability, comparative accuracy and comparative computing time of three explicit numerical solutions of the heat equation for a wall composed of two materials.

2. System of Equations. The equation for the one-dimensional flow of heat is:

$$
c_{s} \rho_{s} \frac{\partial u}{\partial t}=\frac{\partial}{\partial x}\left(k_{s} \frac{\partial u}{\partial x}\right)
$$

$$
\text { where } \begin{aligned}
a_{s-1} & \leqq x \leqq a_{s} \quad s=1,2 \\
0 & <t \leqq t_{F}
\end{aligned}
$$

with the condition at the interface

$$
k_{1}\left(\frac{\partial u}{\partial x}\right)_{1}=k_{2}\left(\frac{\partial u}{\partial x}\right)_{2}
$$

where $\rho_{s}, c_{s}$, and $k_{s}$ are constant with respect to time and temperature but may be different for each material.

We will assume the boundary conditions:

$$
\begin{aligned}
& u\left(a_{0}, t\right)=\text { constant }_{1} t \geqq 0 \\
& u\left(a_{2}, t\right)=\text { constant }_{2} t \geqq 0
\end{aligned}
$$

and initial conditions:

$$
u(x, 0)=\text { constant }_{3} a_{0}<x<a_{2}
$$

Let each material's thickness, $a_{s}-a_{s-1}$, be divided into $N_{s}$ equal parts of $\Delta x_{s}$, and $t_{F}$ into equal parts of $\Delta t_{s}$. Let $i$ denote the subscript associated with the space variable and $j$ the subscript associated with the time variable. Let the solution of (1)-(4) be called $T(x, t)$.

Taylor series expansions of $T_{i j+1}, T_{i+1, j}$, and $T_{i-1, j}$, about $T_{i j}$ are used to obtain

$$
T_{i, j+1}=\frac{r_{s} \Delta t}{\Delta x_{s}^{2}}\left[T_{i+1, j}-2 T_{i j}+T_{i-1, j}\right]+T_{i j}+E_{1}
$$

where $r_{s}=k_{s} / \rho_{s} c_{s}$ and

$$
E_{1}=\frac{\Delta t^{2}}{2} \frac{\partial^{2} T}{\partial t^{2}}-\frac{r_{s} \Delta t \Delta x_{s}{ }^{2}}{12} \frac{\partial^{4} T}{\partial x^{4}}+\text { terms of higher order. }
$$

Omitting $E_{1}$, equation (5) gives a difference equation for finding the approximate

Received December 28, 1959; revised April 27, 1960. 
solution of (1)-(4), $T_{i, j+1}$, when $x_{i}, x_{i-1}$, and $x_{i+1}$ are in the same material. This is the same as the forward difference equation for a one-material wall.

The following equation for $T_{i, j+1}$ at the interface is derived in a manner similar to that used by M. Lotkin [5] in his discussion of an implicit method for a wall of two materials.

$$
T_{i j}+\frac{\left[\left(T_{i+1, j}-T_{i j}\right) \frac{k_{2}}{\Delta x_{2}}+\left(T_{i-1, j}-T_{i j}\right) \frac{k_{1}}{\Delta x_{1}}\right] 2 \Delta t}{\Delta x_{2} c_{2} \rho_{2}+\Delta x_{1} c_{1} \rho_{1}}=T_{i, j+1}+E_{2}
$$

where

$$
\begin{aligned}
E_{2}=\frac{2 \Delta t}{\Delta x_{2} c_{2} \rho_{2}+\Delta x_{1} c_{1} \rho_{1}}- & {\left[\frac{\Delta x_{1} c_{1} \rho_{1} \Delta t}{4} \frac{\partial^{2} T}{\partial t^{2}}-\frac{\Delta x_{1}{ }^{2} k_{1}}{6}\left(\frac{\partial^{3} T}{\partial x^{3}}\right)_{1}\right.} \\
+ & \frac{k_{1} \Delta x_{1}^{3}}{24}\left(\frac{\partial^{4} T}{\partial x^{4}}\right)_{1}-\frac{\Delta x_{2} c_{2} \rho_{2} \Delta t}{4} \frac{\partial^{2} T}{\partial t^{2}} \\
+ & \left.\frac{\Delta x_{2}^{2} k_{2}}{6}\left(\frac{\partial^{3} T}{\partial x^{3}}\right)_{2}+\frac{k_{2} \Delta x_{2}^{3}}{24}\left(\frac{\partial^{4} T}{\partial x^{4}}\right)_{2}\right]+ \text { terms of higher order. }
\end{aligned}
$$

Omitting $E_{2}$, equation (6) gives a difference equation for finding $T_{i, j+1}$ when $x_{1}$ is at the interface, $x_{i-1}$ is in the first material, and $x_{i+1}$ is in the second material.

3. Definition of Methods. Stability is maintained in the explicit numerical solution for a wall of one material by choosing

$$
\Delta t \leqq \frac{\rho c \Delta x^{2}}{2 k} .
$$

Using equations (5) and (6) as our basic computing equations, three different means of choosing $\Delta t$ will be defined and thereby different computational schemes. We will arbitrarily assume that $r_{1}>r_{2}$ and, for simplicity, will confine the discussion to cases in which $r_{1} / r_{2}$ is an integer $R$, and $\sqrt{r_{1} / r_{2}}$ is an integer.

If $\Delta x$ is specified as the thickness of each lamina within the wall, equation (7) gives two different maximum usable time increments depending on the properties of each material;

$$
\Delta t_{1}=\frac{\Delta x^{2}}{2 r_{1}} \text { and } \Delta t_{2}=\frac{\Delta x^{2}}{2 r_{2}} .
$$

METhod 1. In the first method an attempt is made to circumvent the difficulty of having two $\Delta t$ 's by letting $\Delta x_{1}=\Delta x$ and redefining $\Delta x_{2}$ such that $\Delta x_{2}=\Delta x_{1} / \sqrt{R}$. This increases the number of laminae in the second material but yields only one time increment, $\Delta t_{1}=\Delta t_{2}=\Delta t$. The computations would take place as follows:

Given $\Delta x=\Delta x_{1}, r_{1}$ and $r_{2}$

1. Compute $\Delta x_{2}=\Delta x_{1} / \sqrt{R}$

2. Find $\Delta t=\Delta t_{1}=\Delta t_{2}$

3. Set time equal to $\Delta t$

4. Use equation (5) to find the temperatures in material 1.

5. Use equation (6) to find the interface temperature.

6. Use equation (5) to find the temperatures in material 2. 
7. Advance the time by $\Delta t$.

8. Repeat steps 4 to 7 until the temperatures at $t_{F}$ have been computed.

METHoD 2. In the second method $\Delta t$ is chosen by evaluating $\Delta t_{1}$ and $\Delta t_{2}$ and using whichever is less. The computations would then be:

Given $\Delta x=\Delta x_{1}=\Delta x_{2}, r_{1}$ and $r_{2}$

1. Choose $\Delta t=\min \left(\Delta t_{1}, \Delta t_{2}\right)$

2. Proceed as in steps 3 to 8 of Method 1.

MeтноD 3. In the third method both time increments are utilized by using the smaller increment only for those points at which it is necessary and the larger increment for the rest. The smaller time increment must be used for all points in the first material, at the interface, and for enough points in the second material to enable a smooth transition. For example, when $R=4$, those points on the grid in Figure 1 denoted by dots are computed using $\Delta t_{1}$ and then those denoted by crosses are computed using $\Delta t_{2}$. The computations would proceed as follows:

Given $\Delta x=\Delta x_{1}=\Delta x_{2}, r_{1}$ and $r_{2}$

1. Compute $\Delta t_{1}$

2. Compute $\Delta t_{2}$

3. Set time $=0$

4. Set $Q=R-1$

5. Advance time by $\Delta t_{1}$

6. Compute the temperatures in material 1 using equation (5) and $\Delta t_{1}$.

7. Compute the interface temperature using equation (6) and $\Delta t_{1}$.

8. If $Q=0$ proceed to step 12 .

9. Compute $Q$ points in material 2 using equation (5) and $\Delta t_{1}$.

10. $Q-1 \rightarrow Q$.

11. Repeat steps 5 to 10 until indicated by step 8 .

12. Compute temperatures in material 2 using equation (5) and $\Delta t_{2}$.

13. Repeat steps 4 to 12 until the temperatures at $t_{F}$ have been computed.

\section{Convergence of Solutions.}

THEOREM. If there exists a solution of the system of equations (1) to (4) which has bounded derivatives $\partial^{2} T / \partial t^{2}, \partial^{3} T / \partial x^{3}$, and $\partial^{4} T / \partial x^{4}$ in $0 \leqq t \leqq t_{F}, a_{0} \leqq x<a_{1}$ and

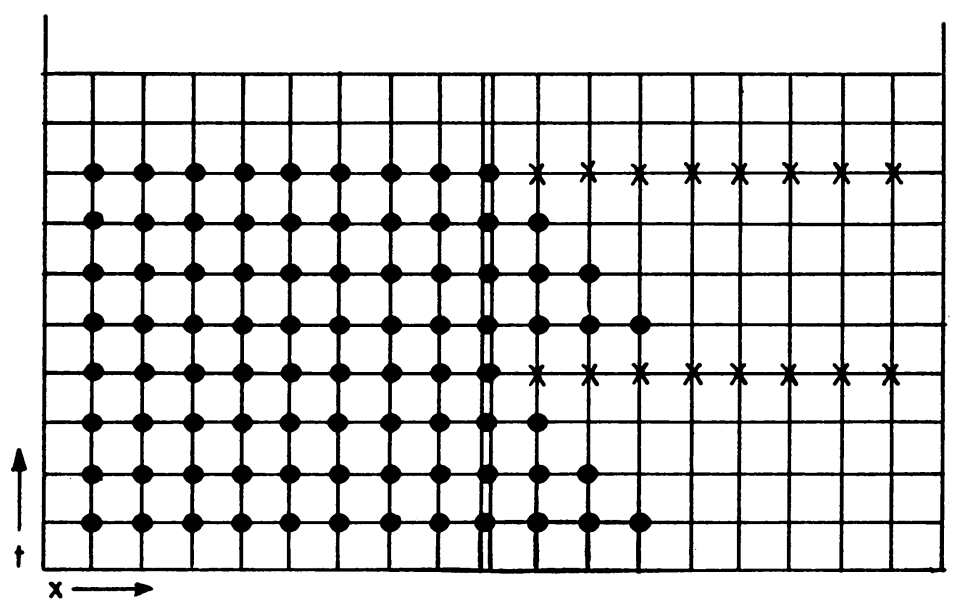

Fig. 1. - Grid points for $R=4$. 
$a_{1}<x \leqq a_{2}$, then the solutions obtained with methods 1,2 , and 3 converge to the true solution. The rate of convergence is $O\left(\Delta x^{2}\right)$.

Proof. Let $B_{1}=\mid$ upper bound on $\partial^{2} T / \partial t^{2}\left|, B_{2}=\right|$ upper bound on $\partial^{4} T / \partial x^{4} \mid$, and $B_{3}=\mid$ upper bound on $\partial^{3} T / \partial x^{3} \mid$. A barred derivative denotes that it is evaluated somewhere within the interval $0 \leqq t \leqq t_{F}, a_{0} \leqq x<a_{1}, a_{1}<x \leqq a_{2}$. Define the error at the point $x_{i}, t_{j}$ to be $e_{i j}=T_{i j}-u_{i j}$. Here $u_{i j}$ is the true solution of (1)-(4). The error arising from the use of equation (5) satisfies the following equation:

$$
\begin{aligned}
& e_{i, j+1}=\frac{r_{s} \Delta t}{\Delta x_{s}^{2}}\left[e_{i+1, j}-2 e_{i j}+e_{i-1, j}\right]+e_{i j}+\Delta t^{2} \frac{\overline{\partial^{2} T}}{\partial t^{2}}-\frac{r_{s} \Delta t \Delta x_{s}{ }^{2} \overline{\partial^{4} T}}{12} \\
& e_{i, j+1} \leqq\left|\frac{r_{8} \Delta t}{\Delta x_{8}{ }^{2}}\right| e_{i+1, j}+\left|1-\frac{2 r_{8} \Delta t}{\Delta x_{s}{ }^{2}}\right| e_{i j}+\left|\frac{r_{8} \Delta t}{\Delta x_{s}{ }^{2}}\right| e_{i-1, j}+\frac{\Delta t^{2}}{2} B_{1}+\frac{r_{8} \Delta t \Delta x_{s}^{2}}{12} B_{2}
\end{aligned}
$$

Let $\alpha_{j}=\max _{i}\left|e_{i j}\right|$, then

$$
e_{i, j+1} \leqq\left[\left|\frac{r_{s} \Delta t}{\Delta x_{s}{ }^{2}}\right|+\left|1-\frac{2 r_{s} \Delta t}{\Delta x_{s}{ }^{2}}\right|+\left|\frac{r_{s} \Delta t}{\Delta x_{s}{ }^{2}}\right|\right] \alpha_{j}+\frac{\Delta t^{2}}{2} B_{1}+\frac{r_{s} \Delta t \Delta x_{s}{ }^{2}}{12} B_{2} .
$$

In methods 1 and $2, \Delta t \leqq \Delta x_{s}{ }^{2} / 2 r_{s}(s=1,2)$ and therefore $r_{s} \Delta t / \Delta x_{s}{ }^{2} \leqq \frac{1}{2}$. This causes each of the terms within the absolute value signs in equation $\left(8^{\prime \prime}\right)$ to be positive and so they may be eliminated giving

$$
e_{i, j+1} \leqq \alpha_{j}+\frac{\Delta t^{2}}{2} B_{1}+\frac{\Delta x_{s}{ }^{4}}{24} B_{2} .
$$

In method 3 , the larger time increment, $\Delta t_{2}$, is only used at points for which $\Delta t_{2} \leqq \Delta x_{2}{ }^{2} / 2 r_{2}$ is satisfied and whenever $\Delta t_{1}$ is used, in the first material or for the transition values, $\Delta t_{1} \leqq \Delta x_{s}{ }^{2} / 2 r_{s}(s=1,2)$. Therefore, whenever equation (5) is used $\Delta t \leqq \Delta x^{2} / 2 r$ and so equation (9) also applies to method 3 .

The error in the evaluation of the interface temperature from equation (6) satisfies

$$
\begin{aligned}
e_{i, j+1}= & e_{i, j}+\frac{\left[\left(e_{i+1, j}-e_{i j}\right) \frac{k_{2}}{\Delta x_{2}}+\left(e_{i-1, j}-e_{i j}\right) \frac{k_{1}}{\Delta x_{1}}\right] 2 \Delta t}{\Delta x_{2} c_{2} \rho_{2}+\Delta x_{1} c_{1} \rho_{1}}-E_{2} \\
e_{i, j+1} \leqq \alpha_{j} & {\left[\frac{k_{2}}{\Delta x_{2}}+\frac{k_{1}}{\Delta x_{1}}\right]\left[\frac{2 \Delta t}{\Delta x_{2} c_{2} \rho_{2}+\Delta x_{1} c_{1} \rho_{1}}\right] } \\
& +\left|1-\frac{2 \Delta t\left[\frac{k_{2}}{\Delta x_{2}}+\frac{k_{1}}{\Delta x_{1}}\right]}{\Delta x_{2} c_{2} \rho_{2}+\Delta x_{1} c_{1} \rho_{1}}\right| \alpha_{j}+\frac{\Delta t^{2}}{2} B_{1}+\frac{\Delta t B_{3}\left(\Delta x_{1}{ }^{2} k_{1}+\Delta x_{2}{ }^{2} k_{2}\right)}{3\left(\Delta x_{2} c_{2} \rho_{2}+\Delta x_{1} c_{1} \rho_{1}\right)} \\
& +\frac{\Delta t B_{2}\left(k_{1} \Delta x_{1}{ }^{3}+k_{2} \Delta_{2}^{3}\right)}{12\left(\Delta x_{2} c_{2} \rho_{2}+\Delta x_{1} c_{1} \rho_{1}\right)} .
\end{aligned}
$$

Since in all three methods the $\Delta t$ used in the interface equation is $\min \left(\Delta t_{1}, \Delta t_{2}\right)$,

$$
\frac{\Delta x_{2} c_{2} \rho_{2}}{\Delta t}+\frac{\Delta x_{1} c_{1} \rho_{1}}{\Delta t} \geqq 2\left[\frac{k_{2}}{\Delta x_{2}}+\frac{k_{1}}{\Delta x_{1}}\right]
$$


and, therefore, (11) can be rewritten as

$$
\begin{aligned}
e_{i, j+1} \leqq \alpha_{j}+\frac{\Delta t^{2}}{2} B_{1}+\frac{B_{3}}{6} & {\left[\frac{k_{1} \Delta x_{2}\left(\Delta x_{1}{ }^{3}\right)+k_{2} \Delta x_{1}\left(\Delta x_{2}{ }^{3}\right)}{\Delta x_{1} k_{2}+\Delta x_{2} k_{1}}\right] } \\
+\frac{B_{2}}{24} & {\left[\frac{k_{1} \Delta x_{2}\left(\Delta x_{1}{ }^{4}\right)+k_{2} \Delta x_{1}\left(\Delta x_{2}{ }^{4}\right)}{\Delta x_{1} k_{2}+\Delta x_{2} k_{1}}\right] . }
\end{aligned}
$$

Let $\Delta x=\max \left(\Delta x_{1}, \Delta x_{2}\right)$, then (12) becomes

$$
e_{i, j+1} \leqq \alpha_{j}+\frac{\Delta t^{2}}{2} B_{1}+\frac{\Delta x^{3}}{6} B_{3}+\frac{\Delta x^{4}}{24} B_{2}
$$

Comparing equations (9) and (13), it can be seen that

(14) $\alpha_{j+1} \leqq \alpha_{j}+\frac{\Delta t^{2}}{2} B_{1}+\frac{\Delta x^{3}}{6} B_{3}+\frac{\Delta x^{4}}{24} B_{2}$ where $\Delta t=\max \left(\Delta t_{1}, \Delta t_{2}\right)$

and $\Delta x=\max \left(\Delta x_{1}, \Delta x_{2}\right)$. At any point $t=j \Delta t$

$$
\alpha_{j} \leqq \alpha_{0}+j\left[\frac{\Delta t^{2}}{2} B_{1}+\frac{\Delta x^{3}}{6} B_{3}+\frac{\Delta x^{4}}{24} B_{2}\right] .
$$

The rate of convergence is, therefore, of the order $0\left(\Delta t \& \Delta x^{2}\right)$. Since $\Delta t$ is of the order $0\left(\Delta x^{2}\right)$, the rate of convergence is $0\left(\Delta x^{2}\right)$.

5. Analytical Example. In order to examine the performance of the three methods, a test case will be used for which some analytical solutions are known. The equations for the composite wall will first be reduced, by transformations of the variables, to the equations for a wall of one material. To do this we will impose the conditions:

$$
\begin{aligned}
k_{1} c_{1} \rho_{1} & =k_{2} c_{2} \rho_{2} \\
a_{0} & =0 .
\end{aligned}
$$

Define the transformations

$$
\begin{gathered}
y=\left\{\begin{array}{lc}
{\left[b+(1-b) \frac{a_{2}}{a_{1}}\right] x} & 0 \leqq x \leqq a_{1} \\
b x+(1-b) a_{2} & a_{1} \leqq x \leqq a_{2}
\end{array}\right. \\
\begin{array}{rl}
\tau=r_{2} b^{2} t & 0 \leqq t \leqq t_{7}
\end{array} \\
\text { where } b=\frac{k_{1} a_{2}}{k_{1}\left(a_{2}-a_{1}\right)+k_{2} a_{1}} .
\end{gathered}
$$

This reduces equations (1) to (4) to

$$
\begin{gathered}
\frac{\partial u}{\partial \tau}=\frac{\partial^{2} u}{\partial y^{2}} \quad 0 \leqq y \leqq a_{2}, 0 \leqq \tau \leqq t_{F} r_{2} b^{2} \\
u(0, \tau)=\text { constant }_{1} \tau \geqq 0 \\
u\left(a_{2}, \tau\right)=\text { constant }_{2} \tau \geqq 0 \\
u(y, 0)=\text { constant }_{3} 0<y<a_{2} .
\end{gathered}
$$


The solution to this set of equations is found in reference [6] for $\tau=0(.005) .1$, $y=.4$, constant $1=$ constant $_{2}=0$, constant $_{3}=1, a_{2}=1$.

6. Test Case Results. Each method was programmed for the IBM 704 EDPM. The test data used was $k_{1}=10, c_{1}=5, \rho_{1}=2, k_{2}=5, c_{2}=4, \rho_{2}=5, a_{0}=0$, $a_{1}=.5$, and $a_{2}=1$. Each method was run for three cases: Case A, $\Delta x=.1$; case $\mathrm{B}, \Delta x=.05$, and case $\mathrm{C}, \Delta x=.025$.

To examine the rate of convergence, the maximum differences for a given time point were found between the results for case $A$ and case $C$, and the results for case $B$ and case $C$. The ratios of these maximum differences ranged between 3.8 and 5.0 for each of the three methods. Since the ratios of the $\Delta x$ 's were 2.0, this would seem to corroborate that the rate of convergence is $0\left(\Delta x^{2}\right)$.

To compare the accuracy of the three methods, the data presented in reference [6] was used. Their values correspond, according to the transformation presented in equation (17), to $x=.55$ and $x=.7$ for $t=0(.01125) .225$. These values, as well as those obtained for case $\mathrm{C}$ for the three methods, are presented in Table 1. It can be seen from the table that, although they all showed close agreement, method 2 gave the most accuracy with a maximum of $.08 \%$ error, method 1 the next with a maximum of $.14 \%$ error, and method 3 the least with a maximum of $.28 \%$ error. The symmetry of the transformed equation indicates that for this case the temperatures should be the same for $x=.15$ and $x=.925, x=.3$ and $x=.85, x=.45$ and $x=.775$, and for $x=.55$ and $x=.7$. When comparing the results at these points at $t=.1$, method 1 has them all the same, method 2 has a greatest difference of $.04 \%$, and method 3 has a greatest difference of $.53 \%$. A comparison at $t=.225$ shows method 1 has them all the same, method 2 still with a greatest difference of $.04 \%$, and method 3 with a greatest difference of $.20 \%$. These differences are reasonable in terms of the methods of choosing $\Delta t$ for computation and illustrate that in method 1 the results for both materials is equally accurate, for method 2 the results for the second material is a bit more accurate than for the first material, while for method 3 the result for the second material is less accurate than for the first but their differences decrease as more time steps are taken.

Table 1

\begin{tabular}{c|c|c|c|c|c|c|c}
\hline Time & $\begin{array}{c}\text { Exact } \\
\text { Solution }\end{array}$ & $\begin{array}{c}\text { Method 1, } \\
x=.55\end{array}$ & $\begin{array}{c}\text { Method 1. } \\
x=.7\end{array}$ & $\begin{array}{c}\text { Method 2, } \\
x=.55\end{array}$ & $\begin{array}{c}\text { Method 2, } \\
x=.7\end{array}$ & $\begin{array}{c}\text { Method 3, } \\
x=.55\end{array}$ & $\begin{array}{c}\text { Method 3, } \\
x=.7\end{array}$ \\
\cline { 2 - 7 } .0225 & .9953 & .99536 & .99536 & .99551 & .99499 & .99579 & .99557 \\
.0450 & .9518 & .95145 & .95145 & .95188 & .95127 & .95254 & .95055 \\
.0675 & .8832 & .88261 & .88261 & .88306 & .88275 & .88354 & .88136 \\
.0900 & .8088 & .80807 & .80807 & .80851 & .80840 & .80868 & .80686 \\
.1125 & .7363 & .73556 & .73556 & .73598 & .73597 & .73590 & .73448 \\
.1350 & .6686 & .66782 & .66782 & .66821 & .66826 & .66797 & .66689 \\
.1575 & .6063 & .60561 & .60561 & .60597 & .60605 & .60562 & .60481 \\
.1800 & .5496 & .54891 & .54891 & .54924 & .54933 & .54882 & .54821 \\
.2025 & .4981 & .49739 & .49739 & .49770 & .49780 & .49724 & .49678 \\
.2250 & .4513 & .45067 & .45067 & .45095 & .45105 & .45047 & .45011 \\
\hline
\end{tabular}


Although the time increments were chosen on the basis of equation ( 7 ), it is pointed out in reference [6] that a slightly larger increment is possible, namely

$$
\Delta t_{\max } \leqq \frac{c \rho \Delta x^{2}}{2 k \sin ^{2}\left[\frac{(N-1) \pi}{2 N}\right]}
$$

Using case $\mathbf{A}$, the programs were run until instability appeared in an attempt to see what the maximum increment actually was for two materials. For method 3, if the maximum $\Delta t$ 's are computed separately for each material of 5 laminae with equation (21), the result is $\Delta t_{1} \leqq .005528$ and $\Delta t_{2} \leqq .022112$. The experimental results corroborated this since it was stable up to $\Delta t_{1}=.0055$ and $\Delta t_{2}=.0220$ but unstable for $\Delta t_{1}=.0056$ and $\Delta t_{2}=.0230$. For method 2 , computing the maximum time increment for each material of 5 laminae and then choosing the smaller, one gets $\Delta t \leqq .005528$. The test showed the same result as it was stable up to $\Delta t=.0055$ but unstable for $\Delta t=.0056$. In method 1 , when using equation ( 7 ), the adjustment of the thicknesses of the laminae in the second material led to equal $\Delta t$ 's. However, when using equation (21), the $\Delta t$ 's obtained are $\Delta t_{1} \leqq .005528$ and $\Delta t_{2} \leqq .005125$ since the number of laminae in each material is different. The test runs showed that it remained stable until $\Delta t=.0053$ but was unstable with $\Delta t=.0054$. This might indicate that a maximum time increment was used which is the average of $\Delta t_{1}$ and $\Delta t_{2}$ but no conclusion is possible since the stability condition states that it should be stable below the computed $\Delta t_{\max }$ but it is not necessarily unstable for a $\Delta t$ above it. However, all these stability test runs seem to indicate that when there are two materials in a wall, the maximum usable time increment is quite closely related to the maximum increments computed for each material separately.

7. Comparison of Computing Time Required. The amount of computing time required for each method can be compared by comparing the number of temperatures that must be evaluated.

Let us assume that the thicknesses of the first and second materials are equal and that $t_{F}=P R \Delta t_{1}$ (where $P$ is any integer). It should be noted that given $\Delta x$, $r_{1}$, and $r_{2}$, each method will compute the same value for $\Delta t_{1}$ and $\Delta x_{1}$.

In method 1 there are $N_{1}(1+\sqrt{ } \bar{R})$ laminae and $P R$ time points. Therefore, the number of temperatures computed equals $P\left[R N_{1}(1+\sqrt{R})-R\right]$. In method 2 there are $2 N_{1}$ laminae and $P R$ time points and so $P\left[2 N_{1} R-R\right]$ temperatures are computed. In method 3 each of the $N_{1}-1$ temperatures in the first material

TABLE 2

\begin{tabular}{c|c|c|c}
\hline & Case A & Case B & Case C \\
\hline Method 1 & $\mathrm{P}(56)$ & $\mathrm{P}(116)$ & $\mathrm{P}(236)$ \\
Method 2 & $\mathrm{P}(36)$ & $\mathrm{P}(76)$ & $\mathrm{P}(156)$ \\
Method 3 & $\mathrm{P}(30)$ & $\mathrm{P}(55)$ & \\
\hline $\begin{array}{c}\text { Ratio } \\
\text { M1:M2:M3 }\end{array}$ & $1.87: 1.20: 1.00$ & $2.11: 1.38: 1.00$ & $2.25: 1.49: 1.00$ \\
\hline
\end{tabular}


is computed $P R$ times, the interface is computed $P R$ times, and for each step of $\Delta t_{2}$, which occurs $P$ times, $N_{1}-1$ values are computed plus the additional number of transition values $=\sum_{i=1}^{R-1} i$. Method 3 , therefore, computes

$$
P\left[N_{1} R+N_{1}-1+\frac{R^{2}}{2}-\frac{R}{2}\right]
$$

values. From this it can be seen that for all $R>1$ method 2 is faster than method 1 . For $2 N_{1}-2>R>1$ method 3 is faster than method 2. This comparison is illustrated in Table 2 for cases $\mathrm{A}, \mathrm{B}$ and $\mathrm{C}$ of the test data used.

8. Conclusions. The analysis and test cases used considered constant boundary and initial conditions. Since the stability and convergence depend also on the boundary and initial conditions, as has been pointed out in references [1], [3], and [4], it is quite possible that the introduction of varying conditions would lead to different results as to the usefulness of each method.

From this study it seems that method 1 is the least acceptable since it takes the most computing time, gives less accuracy than method 2 , and presents the most difficulty when $R$ is not an integer. Depending on the amount of accuracy desired, methods 2 and 3 seem of equal usefulness since method 2 gives the most accuracy but method 3 takes less computing time.

9. Acknowledgements. The author wishes to thank Dr. Peter Henrici for his suggestions concerning this study.

Douglas Aircraft Company

Santa Monica, California

1. G. W. Evans, R. Brousseau \& R. Keirstead, "Stability considerations for various difference equations derived for the heat conduction equation," Jn. Math. Phys., v. 34, 1956, p. 267-285.

2. F. B. HILDEBRAND, "On the convergence of numerical solutions of the heat flow equation," Jn. Math. Phys., v. 31, 1952, p. 35-41.

3. M. L. Juncosa \& D. M. Young, "On the order of convergence of solutions of a difference equation to a solution of the diffusion equation," Jn. Soc. Ind. Appl. Math., v. 1, 1953, p. 111-135.

4. W. LEUTERT, "On the convergence of approximate solutions of the heat equation to the exact solution," Amer. Math. Soc. Proc., v. 2, 1951, p. 433-439.

5. M. LOTRIN, "Numerical integration of heat conduction equations," Jn. Math. Phys., v. 37,1958, p. 178-187.

6. G. G. O'Brien, M. A. Hrman \& S. Kaplan, "A study of the numerical solution of partial differential equations," Jn. Math. Phys., v. 29, 1951, p. 223-251. 